\title{
Erratum to: Understanding ethnic differences in mental health service use for adolescents' internalizing problems: the role of emotional problem identification
}

\author{
Esmée E. Verhulp · Gonneke W. J. M. Stevens • \\ Rens van de Schoot - Wilma A. M. Vollebergh
}

Published online: 7 May 2013

(C) Springer-Verlag Berlin Heidelberg 2013

\section{Erratum to: Eur Child Adolesc Psychiatry DOI 10.1007/s00787-013-0380-3}

In the original publication, internalizing problem symptoms at wave 1 were accidently used in the adolescent model instead of internalizing problem symptoms at wave 2. Therefore, the means and confidence intervals presented in the last row of Table 1 are incorrect.

In the note under Table 1, the sentence, "Overlapping confidence intervals of emotional problem identification in the four groups indicate an absence of differences between the groups" should be changed to "Overlapping confidence intervals of emotional problem identification/internalizing problems in the four groups indicate an absence of differences between the groups". The corrected Table 1 is reproduced in the following page.
In Fig. 2, the correlation between emotional problem identification and internalizing problems among adolescents should be 0.60 instead of 0.41 and there is an effect from Moroccan adolescents to internalizing problem symptoms (path A2). The corrected Fig. 2 is reproduced in the following page.

Furthermore, in the last paragraph of the results, the second sentence (line 4) "Among adolescents, there were also no effects of ethnicity on self-reported internalizing problem symptoms (path A2)." should read as "For Moroccan adolescents a negative effect was found from ethnicity to internalizing problem symptoms ( $\beta=-.15$; path A2)."

Despite these small changes in the results, the conclusions remained similar. For Moroccan adolescents, emotional problem identification mediated the association between ethnicity and mental health service use.

The online version of the original article can be found under doi:10.1007/s00787-013-0380-3.

E. E. Verhulp $(\bowtie)$ · G. W. J. M. Stevens · W. A. M. Vollebergh

Utrecht University, Utrecht Centre of Child and Adolescent

Studies, P.O. Box 80.140, 3508 TC Utrecht, The Netherlands

e-mail: e.e.verhulp@uu.nl

R. van de Schoot

Department of Methods and Statistics, Utrecht University,

Utrecht, The Netherlands

R. van de Schoot

North-West University, Optentia Research Program,

Faculty of Humanities, Vanderbijlpark, South Africa 
Table 1 Percentages of mental health service use, means (95\% confidence intervals) of emotional problem identification and internalizing problem symptoms for the different ethnic groups as reported by parents and adolescents

\begin{tabular}{|c|c|c|c|c|}
\hline & Native Dutch & Surinamese & Turkish & Moroccan \\
\hline \multicolumn{5}{|l|}{ Parents } \\
\hline Mental health care (\%) & 31 & $18^{*}$ & $10 *$ & $7 *$ \\
\hline Problem identification & $3.25(2.77-3.73)$ & $2.13(1.64-2.62)^{*}$ & $1.47(0.99-1.97)^{*}$ & $0.62(0.10-1.13)^{*}$ \\
\hline Internalizing problems & $9.00(7.91-10.22)$ & $7.38(6.31-8.46)$ & $9.13(8.06-10.45)$ & $3.99(2.72-5.25)^{*}$ \\
\hline \multicolumn{5}{|l|}{ Adolescents } \\
\hline Mental health care (\%) & 22 & 18 & 15 & $9 *$ \\
\hline Problem identification & $3.59(3.12-4.06)$ & $2.47(1.98-2.96)^{*}$ & $2.41(1.94-2.91)^{*}$ & $2.13(1.61-2.63)^{*}$ \\
\hline Internalizing problems & $13.58(11.97-15.36)$ & $12.03(10.45-13.62)$ & $13.54(11.97-15.49)$ & $10.58(8.71-12.44)$ \\
\hline
\end{tabular}

Problem identification $=$ emotional problem identification. Overlapping confidence intervals of emotional problem identification/internalizing problems in the four groups indicate an absence of differences between the groups

* Indicates that the specific immigrant group differs from the native Dutch reference group

Fig. 2 Results of the mediation analyses showing the effects separately for parents $(P)$ and adolescents $(A)$, after controlling for age, gender, and education level of parents. Path $A 1$ and $A 2$ show the betas of the effects for the three immigrant groups, respectively,

Surinamese, Turkish, and Moroccan. The bold path indicates a significant indirect effect

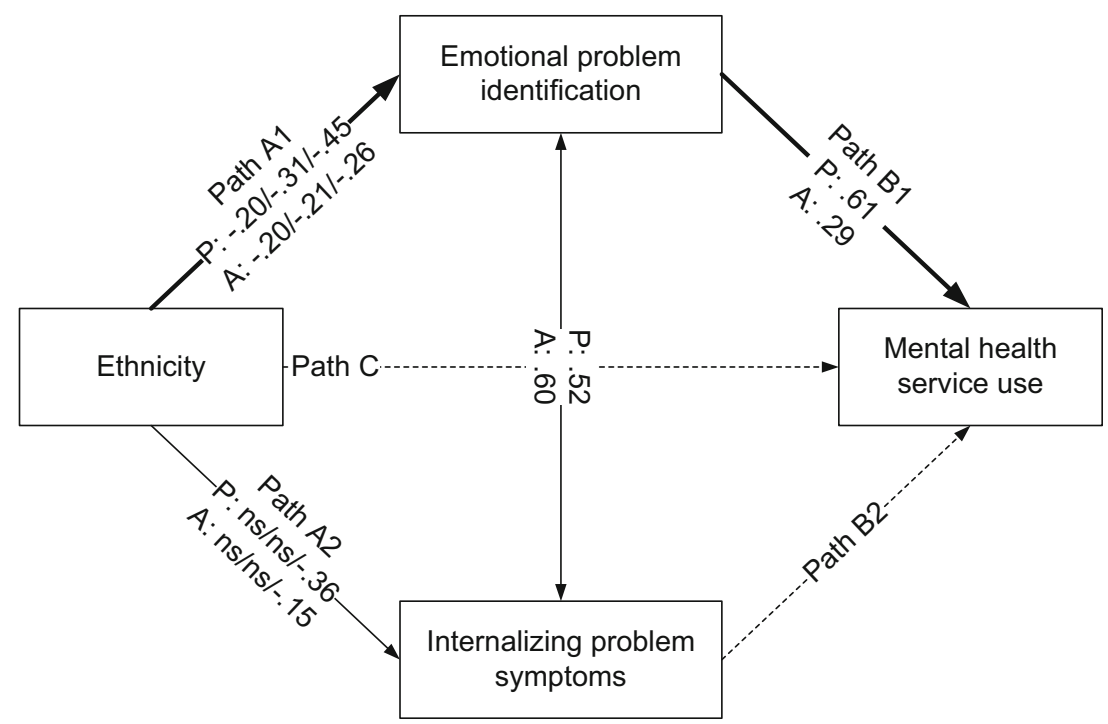

